EDITORIAL EDITORIAL

\section{Endemias e \\ epidemias \\ brasileiras - \\ perspectivas \\ da \\ investigação \\ científica}

Brazilian

endemic and

epidemic

diseases -

prospects of

scientific inves-

tigation

\section{Maria Rita \\ Donalísio}

Editora Especial

Convidada para a série

Endemias/Epidemias

Special Editor invited

for the Endemicl

Epidemic diseases

series
Chronic-degenerative diseases, urban violence, and psychosomatic diseases, to mention just a few, are the conditions that currently have the highest impact on the epidemiological profile of the Brazilian population. Nevertheless, endemic-epidemic transmissible diseases are still a relevant public health problem in Brazil. New diseases or long-known ones (re)appear in different ecological and social contexts. The intervention of health services in complex networks that disseminate infections is limited by macrostructural determinants of the health-disease process, social exclusion, migration, and urban inequality, as highlighted by many authors. However, identifying vulnerable points on the paths of several domestic endemic and epidemic diseases has been one of the main challenges for health professionals, managers and researchers.

We are witnessing the growth in circulating viruses, bacteria and parasites with the (re)emergence of diseases, and their resistance to anti-microbial drugs and insecticides, as well as the adaptation of vectors and $\mathrm{mi}$ croorganisms to new ecological and social scenarios. There is still much to learn about transmissible diseases in the country, even about those that have been widely studied and are traditional public health problems. There is a wide-range of questions to answer, from the molecular intimacy of biological processes to the assessment of national policies for disease control.

Different approaches on disease occurrence and dissemination enrich debate. Studies on epidemic trends and behaviors help predict risk situations. New technologies for early detection and identification of strains ofmicroorganisms explain the paths of transmission better. More powerful and specific medication and immunotherapeutic agents may determine successful control of endemic and epidemic diseases in the country. Multidisciplinary approaches that transcend the scope of Epidemiology contribute to the impact of intervention, particularly in the fields of education on health and community participation. The review of past campaigns, their mistakes and successes, in ad- 
dition to the constant follow-up of current campaigns, encompass the technical challenges of National Public Health technicians.

These investigations often draw new guidelines for health service routines, enabling the incorporation of knowledge and access to experiences in other regions, in addition to facilitating the integration of civil society and its co-responsibility on detecting epidemics.

Although these are fields of research that aim at varied methodological universes, it is in Public Health that these themes fit better, reinforcing the area's vocation in privileging the collective and welcoming different knowledge to explain the health-sickness process in populations.

From this perspective, as of this issue of the Brazilian Journal of Epidemiology we will publish articles on some of the country's most relevant endemic-epidemic transmissible diseases, including: dengue fever, yellow fever, leprosy, influenza, emerging diseases, aids, tuberculosis, meningococcal disease, viral hepatitis, and malaria. The objective is to approach the current limits of scientific investigation, under the prospect of transmission control in the country and to point out the main open issues and promisinglines of research.

In the past decade, we witnessed progressive integration of disease surveillance and control program managers with research groups, and of the latter with services. Would that mean higher commitment of researchers and national financers? Maturity of disease surveillance and control managers searching for concrete solutions? The growing health services public entering postgraduate, senso lato courses, whose institutional ties facilitate commitment with such research themes? Better technical training of human resources in public health, facilitating critical review and flexibility for national/state/municipal programs? Higher demand from the civil society, (better) organized to solve epidemiological problems?

One of the types of research that has been developed in the country is operational research, which can respond faster to every- 
day problems of disease surveillance and control teams. Also, the availability of national databases, whose quality and reliability are increasing (SINAN, SIM, SINASC, SIHSUS, among others), provides timely epidemiological diagnoses.

The association between research interests and those of the health services has generated integrated, light, and cheaper investigation projects, yet conducted with methodological strictness and discipline. In tune with issues of health services, studies come out directed not only to academic libraries, but to fieldwork manuals as well. In this fashion, we enlarge the dialogue between health technicians and researchers, consequently qualifying protocol review, and facilitating the experience of new solutions and technologies that are suitable to national problems. 